Hinchingbrooke Hospital opened at the end of 1983 as a new unit in a new health district. Our obstetric and gynaecological staff comprise four consultants and four general practice trainee senior house officers, and in 1987 we had 2008 deliveries. Like Mr Samra's unit, we have the lowest perinatal mortality rate in our region, $5 \cdot 2$ per 1000 (uncorrected) based on the 7015 deliveries in 1984-7. Neonatal intensive care is available on site, also "two tier"; we do not transfer high risk cases elsewhere. In these four years our caesarean section rate was $13 \cdot 7 \%, 6 \%$ elective and $7 \cdot 7 \%$ emergency; our vaginal instrumental delivery rate was $5 \cdot 2 \%$. Thus it appears that, neglecting possible population differences, we plan rather more elective caesarean sections than are planned in Mr Samra's unit but perform proportionately less than half the number of instrumental deliveries, probably because, without intermediate grade medical staff, our midwives enjoy greater freedom of practice.

No one would suggest that all three tier units should be dismantled immediately and replaced by two tier units, but even in its authors' wildest dreams "Achieving a Balance" cannot live up to its title. Under this plan over half the service posts in the intermediate grade in obstetrics and gynaecology will need to be filled by overseas graduates, brought over usually from developing countries simply to do this work. No other profession runs itself in this way; if a similar staffing structure were suggested for lawyers, teachers, or even general practitioners we as consumers would be horrified. If half Britain's obstetric units converted to two tier working we would be "in balance," with a sound career structure to offer to our aspiring juniors. Overseas visitors could then be treated correctly on a supernumerary basis, as pupils to be taught rather than cheap labour to be exploited.

Certainly, everything in the two tier garden is not fine; much bargaining is still to be done on matters such as staffing levels, free time, and easing of duties once the mid-50s are reached. But we have built a system that is liked by the doctors who work it, the nurses and midwives who support them, and the women who deliver under their care. What we need is for the BMA and the royal colleges to help us improve this system and refine it into an acceptable alternative to three tier working, rather than to pull it down and destroy it.

Hinchingbrooke Hospital,

M J HARE

Huntingdon, Cambridgeshire PE18 8NT

\section{Diagnosis of diabetes mellitus: pitfalls in the glucose tolerance test}

SIR,-Dr K Wiener (28 November, p 1363) draws attention to the World Health Organisation's criteria for diagnosing diabetes mellitus and raises several important issues which appear to remain unresolved despite the latest recommendations of a study group. ${ }^{1}$

The blood glucose response to a glucose load will be influenced by the timing, size, composition, and quantity of preceding food. These influences will be minimised by a suitable period of fasting. No study, however, has adequately examined the influence of the duration of fasting. It therefore seems reasonable to standardise to $10-16$ hours, as recommended by the study group. ${ }^{1}$ The test should be performed in the morning both for convenience and to avoid the effects of circadian variation in glucose tolerance. ${ }^{2}$

All laboratories should standardise the glucose load to $75 \mathrm{~g}$ (or $1.75 \mathrm{~g} / \mathrm{kg}$ body weight to a maximum of $75 \mathrm{~g}$ for children) as recommended by the World Health Organisation ${ }^{1}$ and other authoritative bodies $^{3.5}$ and as emphasised by
Dr Wiener. With regard to anhydrous glucose, as opposed to the monohydrated form, most data have been obtained with $75 \mathrm{~g}$ of pure glucose or its equivalent, and this should be adhered to. Thus $82.5 \mathrm{~g}$ of the monohydrate would be required to deliver the 75 g glucose load.

We agree with $\mathrm{Dr}$ Wiener that the misdiagnosis of diabetes mellitus can have serious implications for the person concerned. In a clinical setting the diagnosis must be corroborated by supporting evidence. A lifetime diagnosis of diabetes mellitus should not be made on the basis of a single abnormal test result alone. ${ }^{1}$ The introduction of the intermediate category of impaired glucose tolerance ${ }^{35}$ was, in part, designed to achieve this objective. Nevertheless, such individuals are at substantially higher risk for the subsequent development of diabetes.

In the epidemiological setting rather different considerations apply. The need for rigorous standardisation becomes paramount; even small, but systematic, differences in the test procedure may result in apparently significant, but spurious, differences between populations in glucose tolerance and in the prevalence of diabetes mellitus. Even taking the glucose load over five minutes, rather than 15 minutes, can make a $1 \mathrm{mmol} / \mathrm{l}$ difference in the mean two hour blood glucose concentration. ${ }^{6}$ Of course, if individuals qualifying for the diagnosis of diabetes come to light during epidemiological surveys it is as important to confirm the diagnosis by suitable repeat measurements as it is in a clinical setting.

The World Health Organisation is now preparing a survey manual for the study of diabetes mellitus, which will provide detailed guidance in these matters.

Division of Noncommunicable Diseases,

World Health Organisation,

CH-1211 Geneva 27, Switzerland

H KING

WHO Collaborating Centre for Research and Development in Laboratory Techniques in Diabetes,

Medical School,

Newcastle upon Tyne NE2 4HH

H KEEN

WHO Collaborating Centre for the Study and Control of Long term Complications of Diabetes Mellitus, Guy's Hospital,

\section{London SE1 9RT}

Epidemiology and Field Studies Branch,

P H BENNETT

National Institutes of Health,

Phoenix, Arizona,

USA

1 World Health Organisation. Diabetes mellitus. Geneva: WHO, 1985. (WHO Technical Report Series No 727.)

2 Jarrett RJ. Rhythms in insulin and glucose. In: Kriger D, ed. Endocrine rhythms. New York: Raven Press, 1979:247-58

3 National Diabetes Data Group. Classification and diagnosis of diabetes mellitus and other categories of glucose tolerance. Diabetes 1979;28:1039-57.

4 Keen H, Jarrett RJ, Alberti KGMM. Diabetes mellitus: a new look at diagnostic criteria. Diabetologia 1979;16:283-5.

5 World Health Organisation Expert Committee on Diabetes Mellitus. Second report. Geneva: WHO, 1980. (WHO Technical Report Series No 646.)

6 Heine RJ, Hanning I, Morgan L, Alberti KGMM. The oral glucose tolerance test (OGTT): effect of rate of ingestion of carbohydrate and different carbohydrate preparations. Diabetes Care 1983;6:441-5.

\section{Secretor state of patients with insulin}

dependent or non-insulin dependent diabetes mellitus

SIR,-Dr P J Lamey and colleagues (12 December, p 1563) presented data contradicting ours (24 October, $p$ 1024). The proportion of non-secretors in their control population (33\%) was, however, unusually high for the United Kingdom. As their letter came from a department of oral medicine and pathology I wonder whether the controls were taken from patients referred for investigation of recurrent oral infection. The authors' group works on oral candidiasis, and we have found a significant increase in the proportion of non-secretors among patients referred to the Edinburgh Dental School for investigation of this condition.

The number of patients with diabetes was roughly half the number examined in our study and the number of controls less than a third.

Nevertheless, the same pattern we observed was present in the results they reported: the proportion of non-secretors with type II diabetes was similar to that of the controls and the proportion of nonsecretors with type I diabetes increased.

\section{C BLACKWELL}

Department of Bacteriology,

University Medical School,

Edinburgh EH8 9AG

1 Blackwell CC, Tom FF, Lawrie O, Weir DM, Wray D, Kinane DF. Non-secretion of blood group antigens and susceptibility to oral infections due to $\mathrm{C}$ albicans. 7 Dent Res 1980;65:502.

\section{Logic in medicine: the role of leads}

SIR,-There was a particularly important omission from the Logic in Medicine series (24 October, p 1033): the role of "leads"1 2 or "pivots"3 in medical problem solving. Leads are findings associated with a limited list of diagnostic possibilities, which are used to simplify the solution of a diagnostic problem. All diagnostic methods whether they are based on computers or human reasoning depend on this type of problem reduction.

Sometimes the presenting complaint is a good lead-for example, a lump in the neck. More often the initial possibilities are numerous and the diagnostician must proceed systematically in the hope that a better lead turns up: a systolic thrill would have been a good lead in Professor Fergus McCartney's example (21 November, p 1325). This lead can be discarded in favour of a better one in due course-for example, an ejection systolic murmur. Previous and subsequent evidence can then be used to eliminate ${ }^{12}$ or prune ${ }^{3}$ all but one of the possibilities raised by the best lead. The pruning may be done by using single items of powerful information ${ }^{4}$ or by using weak evidence and Bayes's theorem with the independence assumption (21 November, p 1329) or some other mathematical device (28 November, p 1392).

It must not be forgotten that hypotheticodeductive thought (31 October, p 1108) also occurs in clinical diagnosis. This begins by choosing one diagnostic possibility from the lead. The diagnostic term represents what the doctor imagines may be going on (the hypothetico part). The possibility is chased by looking for findings which are common in the postulated diagnosis but rare in at least one of the others. A number of such items may thus complement each other by eliminating or "refuting," but not usually completely, all but one of the initial possibilities (the deductive part). This chasing of a diagnostic possibility can be done as soon as a good lead is found by looking only for features which confirm and eliminate (they are simultaneous processes in clinical diagnosis). It is often more convenient, however, to keep going systematically and to leave most of the interpretation until the end, especially if it seems that there are going to be many leads leading to a number of coexisting diagnoses.

Formal logic (7 November, p 1195) can be regarded as a special case of reasoning logically with probabilities or frequencies when some of the latter are zero or $100 \% .{ }^{4} \mathrm{It}$ is something along these 